\title{
Multiplicity results for a fractional Kirchhoff equation involving sign-changing weight function
}

\section{Chuanzhi Bai}

\section{"Correspondence:}

czbai8@sohu.com

Department of Mathematics,

Huaiyin Normal University, Huaian,

Jiangsu 223300, P.R. China

\begin{abstract}
In this paper, we prove the existence and multiplicity of solutions for a fractional Kirchhoff equation involving a sign-changing weight function which generalizes the corresponding result of Tsung-fang Wu (Rocky Mt. J. Math. 39:995-1011, 2009). Our main results are based on the method of a Nehari manifold.
\end{abstract}

MSC: $35 J 50 ; 35 J 60 ; 47 G 20$

Keywords: fractional $p$-Laplacian; Kirchhoff type problem; sign-changing weight; Nehari manifold

\section{Introduction}

In this paper, we consider the following fractional elliptic equation with sign-changing weight functions:

$$
\begin{cases}M\left(\int_{\mathbb{R}^{2 N}} \frac{|u(x)-u(y)|^{p}}{|x-y|^{N+s p}} d x d y\right)(-\Delta)_{p}^{s} u=\lambda f(x) u^{q}+g(x) u^{r}, & x \in \Omega, \\ u=0, & x \in \mathbb{R}^{N} \backslash \Omega,\end{cases}
$$

where $\Omega$ is a smooth bounded domain in $\mathbb{R}^{N}, N>2 s, 0<s<1,0 \leq q<1<r<p_{s}^{*}-1$ $\left(p_{s}^{*}=\frac{p N}{N-p s}\right) ; \lambda>0, M(t)=a+b t^{p-1},(-\Delta)_{p}^{s}$ is the fractional $p$-Laplacian operator defined as

$$
(-\Delta)_{p}^{s} u(x)=2 \lim _{\varepsilon \searrow 0} \int_{B_{\varepsilon}(x)^{c}} \frac{|u(x)-u(y)|^{p-2}(u(x)-u(y))}{|x-y|^{N+s p}} d y, \quad x \in \mathbb{R}^{N}
$$

We may assume that the weight functions $f(x)$ and $g(x)$ are as follows:

(H1) $f^{+}=\max \{f, 0\} \not \equiv 0$, and $f \in L^{\mu_{q}}(\Omega)$ where $\mu_{q}=\frac{\mu}{\mu-(q+1)}$ for some $\mu \in\left(q+1, p_{s}^{*}\right)$, with in addition $f(x) \geq 0$ a.e. in $\Omega$ in the case $q=0$;

(H2) $g^{+}=\max \{g, 0\} \not \equiv 0$, and $g \in L^{\nu_{r}}(\Omega)$ where $v_{r}=\frac{v}{v-(r+1)}$ for some $v \in\left(r+1, p_{s}^{*}\right)$.

The fractional Kirchhoff type problems have been studied by many authors in recent years; see [2-6] and references therein. In the subcritical case, Pucci and Saldi in [5] stud-

(c) The Author(s) 2016. This article is distributed under the terms of the Creative Commons Attribution 4.0 International License (http://creativecommons.org/licenses/by/4.0/), which permits unrestricted use, distribution, and reproduction in any medium, provided you give appropriate credit to the original author(s) and the source, provide a link to the Creative Commons license, and indicate if changes were made. 
ied the following Kirchhoff type problem in $\mathbb{R}^{N}$ :

$$
\begin{cases}M\left(\int_{\mathbb{R}^{2 N}} \frac{|u(x)-u(y)|^{p}}{|x-y|^{N+s p}} d x d y\right)(-\Delta)_{p}^{s} u+V(x)|u|^{p-2} u & \\ =\lambda w(x)|u|^{-2} u-h(x)|u|^{r-2} u, & x \in \Omega, \\ u=0, & x \in \mathbb{R}^{N} \backslash \Omega,\end{cases}
$$

with $n>p s, s \in(0,1)$, and they established the existence and multiplicity of entire solutions using variational methods and topological degree theory for the above problem with a real parameter $\lambda$ under the suitable integrability assumptions of the weights $V$, $w$, and $h$. In [7], Mishra and Sreenadh have studied the following Kirchhoff problem with sign-changing weights:

$$
\begin{cases}M\left(\int_{\mathbb{R}^{2 N}} \frac{|u(x)-u(y)|^{p}}{|x-y|^{N+s p}} d x d y\right)(-\Delta)_{p}^{s} u=\lambda f(x)|u|^{q-2} u+|u|^{\alpha-2} u, & x \in \Omega, \\ u=0, & x \in \mathbb{R}^{N} \backslash \Omega,\end{cases}
$$

and they obtained the multiplicity of non-negative solutions in the subcritical case $\alpha<p_{s}^{*}$ by minimizing the energy functional over non-empty decompositions of Nehari manifold.

When $p=2, s=1, a=1$ and $b=0$, problem (1.1) is reduced to the following semilinear elliptic equation:

$$
\begin{cases}-\Delta u=\lambda f(x) u^{q}+g(x) u^{r}, & x \in \Omega \\ u=0, & x \in \partial \Omega\end{cases}
$$

In [1], Wu proved that equation (1.2) involving a sign-changing weight function has at least two solutions by using the Nehari manifold.

Motivated by the above work, in this paper, we investigate the existence and multiplicity of solutions for a fractional Kirchhoff equation (1.1) and extend the main results of Wu [1].

This article is organized as follows. In Section 2, we give some notations and preliminaries. Section 3 is devoted to the proof that problem (1.1) has at least two solutions for $\lambda$ sufficiently small.

\section{Preliminaries}

For any $s \in(0,1), 1<p<\infty$, we define

$$
X=\left\{u \mid u: \mathbb{R}^{N} \rightarrow \mathbb{R} \text { is measurable, }\left.u\right|_{\Omega} \in L^{p}(\Omega) \text {, and } \int_{Q} \frac{|u(x)-u(y)|^{p}}{|x-y|^{n+p s}} d x d y<\infty\right\},
$$

where $Q=\mathbb{R}^{2 N} \backslash(\mathcal{C} \Omega \times \mathcal{C} \Omega)$ with $\mathcal{C} \Omega=\mathbb{R}^{N} \backslash \Omega$. The space $X$ is endowed with the norm defined by

$$
\|u\|_{X}=\|u\|_{L^{p}(\Omega)}+\left(\int_{Q} \frac{|u(x)-u(y)|^{p}}{|x-y|^{n+p s}} d x d y\right)^{1 / p}
$$

The functional space $X_{0}$ denotes the closure of $C_{0}^{\infty}(\Omega)$ in $X$. By [8], the space $X_{0}$ is a Hilbert space with scalar product

$$
\langle u, v\rangle_{X_{0}}=\int_{Q} \frac{|u(x)-u(y)|^{p-1}(v(x)-v(y))}{|x-y|^{n+p s}} d x d y, \quad \forall u, v \in X_{0}
$$


and the norm

$$
\|u\|_{X_{0}}=\left(\int_{Q} \frac{|u(x)-u(y)|^{p}}{|x-y|^{n+p s}} d x d y\right)^{1 / p}
$$

For further details on $X$ and $X_{0}$ and also for their properties, we refer to [8] and the references therein.

Throughout this section, we denote the best Sobolev constant by $S_{l}$ for the embedding of $X_{0}$ into $L^{l}(\Omega)$, which is defined as

$$
S_{l}=\inf _{X_{0} \backslash\{0\}} \frac{\int_{\mathbb{R}^{2 N}} \frac{|u(x)-u(y)|^{p}}{|x-y|^{N+s p}} d x d y}{\left(\int_{\mathbb{R}^{N}}|u|^{l} d x\right)^{\frac{p}{l}}}>0,
$$

where $l \in\left[p, p_{s}^{*}\right]$.

A function $u \in X_{0}$ is a weak solution of problem (1.1) if

$$
\begin{aligned}
& M\left(\int_{Q} \frac{|u(x)-u(y)|^{p}}{|x-y|^{N+s p}} d x d y\right) \int_{Q} \frac{|u(x)-u(y)|^{p-2}(u(x)-u(y))(v(x)-v(y))}{|x-y|^{N+s p}} d x d y \\
& \quad=\lambda \int_{\Omega} f(x)|u|^{q-1} u v d x+\int_{\Omega} g(x)|u|^{r-1} u v d x, \quad \forall v \in X_{0} .
\end{aligned}
$$

Associated with equation (1.1), we consider the energy functional $\mathcal{J}_{\lambda, M}$ in $X_{0}$

$$
\mathcal{J}_{\lambda, M}(u)=\frac{1}{p} \hat{M}\left(\|u\|_{X_{0}}^{p}\right)-\frac{\lambda}{q+1} \int_{\Omega} f|u|^{q+1} d x-\frac{1}{r+1} \int_{\Omega} g|u|^{r+1} d x,
$$

where $\hat{M}(t)=\int_{0}^{t} M(\mu) d \mu$.

It is easy to see that the solutions of equation (1.1) are the critical points of the energy functional $\mathcal{J}_{\lambda, M}$.

The Nehari manifold for $\mathcal{J}_{\lambda, M}$ is defined as

$$
\begin{aligned}
\mathcal{N}_{\lambda, M}(\Omega) & =\left\{u \in X_{0} \backslash\{0\}:\left\langle\mathcal{J}_{\lambda, M}^{\prime}(u), u\right\rangle=0\right\} \\
& =\left\{\left.u \in X_{0} \backslash\{0\}\left|M\left(\|u\|_{X_{0}}^{p}\right)\|u\|_{X_{0}}^{p}-\lambda \int_{\Omega} f\right| u\right|^{q+1} d x-\int_{\Omega} g|u|^{r+1} d x=0\right\} .
\end{aligned}
$$

The Nehari manifold $\mathcal{N}_{\lambda, M}(\Omega)$ is closely linked to the behavior of functions of the form $h_{\lambda, M}: t \rightarrow \mathcal{J}_{\lambda, M}(t u)$ for $t>0$, named fibering maps [9]. If $u \in X_{0}$, we have

$$
\begin{aligned}
& h_{\lambda, M}(t)=\frac{1}{p} \hat{M}\left(t^{p}\|u\|_{X_{0}}^{p}\right)-\lambda \frac{t^{q+1}}{q+1} \int_{\Omega} f|u|^{q+1} d x-\frac{t^{r+1}}{r+1} \int_{\Omega} g|u|^{r+1} d x, \\
& h_{\lambda, M}^{\prime}(t)=t^{p-1} M\left(t^{p}\|u\|_{X_{0}}^{p}\right)\|u\|_{X_{0}}^{p}-\lambda t^{q} \int_{\Omega} f|u|^{q+1} d x-t^{r} \int_{\Omega} g|u|^{r+1} d x,
\end{aligned}
$$

and

$$
\begin{aligned}
h_{\lambda, M}^{\prime \prime}(t)= & (p-1) t^{p-2} M\left(t^{p}\|u\|_{X_{0}}^{p}\right)\|u\|_{X_{0}}^{p}+p t^{2 p-2} M^{\prime}\left(t^{p}\|u\|_{X_{0}}^{p}\right)\|u\|_{X_{0}}^{2 p} \\
& -q \lambda t^{q-1} \int_{\Omega} f|u|^{q+1} d x-r t^{r-1} \int_{\Omega} g|u|^{r+1} d x .
\end{aligned}
$$


Obviously,

$$
\begin{aligned}
t h_{\lambda, M}^{\prime}(t) & =M\left(t^{p}\|u\|_{X_{0}}^{p}\right)\|t u\|_{X_{0}}^{p}-\lambda \int_{\Omega} f|t u|^{q+1} d x-\int_{\Omega} g|t u|^{r+1} d x \\
& =\left\langle\mathcal{J}_{\lambda, M}(t u), t u\right\rangle,
\end{aligned}
$$

which implies that for $u \in X_{0} \backslash\{0\}$ and $t>0, h_{\lambda, M}(t)=0$ if and only if $t u \in \mathcal{N}_{\lambda, M}(\Omega)$, i.e., positive critical points of $h_{\lambda, M}$ correspond to points on the Nehari manifold. In particular, $h_{\lambda, M}(1)=0$ if and only if $u \in \mathcal{N}_{\lambda, M}(\Omega)$. Hence, we define

$$
\begin{aligned}
& \mathcal{N}_{\lambda, M}^{+}(\Omega)=\left\{u \in \mathcal{N}_{\lambda, M}(\Omega): h_{u, M}^{\prime \prime}(1)>0\right\}, \\
& \mathcal{N}_{\lambda, M}^{0}(\Omega)=\left\{u \in \mathcal{N}_{\lambda, M}(\Omega): h_{u, M}^{\prime \prime}(1)=0\right\}, \\
& \mathcal{N}_{\lambda, M}^{-}(\Omega)=\left\{u \in \mathcal{N}_{\lambda, M}(\Omega): h_{u, M}^{\prime \prime}(1)<0\right\} .
\end{aligned}
$$

For each $u \in \mathcal{N}_{\lambda, M}(\Omega)$, we have

$$
\begin{aligned}
h_{\lambda, M}^{\prime \prime}(1)= & (p-1) M\left(\|u\|_{X_{0}}^{p}\right)\|u\|_{X_{0}}^{p}+p M^{\prime}\left(\|u\|_{X_{0}}^{p}\right)\|u\|_{X_{0}}^{2 p} \\
& -q \lambda \int_{\Omega} f|u|^{q+1} d x-r \int_{\Omega} g|u|^{r+1} d x \\
= & (p-r-1) M\left(\|u\|_{X_{0}}^{p}\right)\|u\|_{X_{0}}^{p}+p M^{\prime}\left(\|u\|_{X_{0}}^{p}\right)\|u\|_{X_{0}}^{2 p}-\lambda(q-r) \int_{\Omega} f|u|^{q+1} d x \\
= & (p-q-1) M\left(\|u\|_{X_{0}}^{p}\right)\|u\|_{X_{0}}^{p}+p M^{\prime}\left(\|u\|_{X_{0}}^{p}\right)\|u\|_{X_{0}}^{2 p}-(r-q) \int_{\Omega} g|u|^{r+1} d x .
\end{aligned}
$$

Let $M(t)=a+b t^{p-1}$, where $a>0, b \geq 0$ and $p>1$. If $u \in \mathcal{N}_{\lambda, M}^{0}(\Omega)$, then $h_{\lambda, M}^{\prime \prime}(1)=0$, and we have by (2.1) and (2.2)

$$
\begin{aligned}
& a(p-r-1)\|u\|_{X_{0}}^{p}+b\left(p^{2}-r-1\right)\|u\|_{X_{0}}^{p^{2}}-\lambda(q-r) \int_{\Omega} f|u|^{q+1} d x=0, \\
& a(p-q-1)\|u\|_{X_{0}}^{p}+b\left(p^{2}-q-1\right)\|u\|_{X_{0}}^{p^{2}}-(r-q) \int_{\Omega} g|u|^{r+1} d x=0 .
\end{aligned}
$$

For convenience, we let (H3) $0<q<1, p>1+q$ and $p_{s}^{*}-1>r \begin{cases}>p^{2}-1, & b \neq 0, \\ >p-1, & b=0 .\end{cases}$

Lemma 2.1 If $(\mathrm{H} 1)$ and $(\mathrm{H} 3)$ hold, then the energy functional $\mathcal{J}_{\lambda, M}$ is coercive and bounded below on $\mathcal{N}_{\lambda, M}(\Omega)$.

Proof For $u \in \mathcal{N}_{\lambda, M}(\Omega)$, we have by the Hölder and Sobolev inequalities

$$
\begin{aligned}
\mathcal{J}_{\lambda, M}(u)= & a\left(\frac{1}{p}-\frac{1}{r+1}\right)\|u\|_{X_{0}}^{p}+b\left(\frac{1}{p^{2}}-\frac{1}{r+1}\right)\|u\|_{X_{0}}^{p^{2}} \\
& -\lambda\left(\frac{1}{q+1}-\frac{1}{r+1}\right) \int_{\Omega} f|u|^{q+1} d x \\
= & a\left(\frac{1}{p}-\frac{1}{r+1}\right)\|u\|_{X_{0}}^{p}+b\left(\frac{1}{p^{2}}-\frac{1}{r+1}\right)\|u\|_{X_{0}}^{p^{2}}
\end{aligned}
$$




$$
\begin{aligned}
& -\lambda \frac{r-q}{(q+1)(r+1)} \int_{\Omega} f|u|^{q+1} d x \\
\geq & a\left(\frac{1}{p}-\frac{1}{r+1}\right)\|u\|_{X_{0}}^{p}+b\left(\frac{1}{p^{2}}-\frac{1}{r+1}\right)\|u\|_{X_{0}}^{p^{2}} \\
& -\lambda \frac{r-q}{(q+1)(r+1)}\|f\|_{L^{\mu q}} S_{\mu}^{q+1}\|u\|_{X_{0}}^{q+1},
\end{aligned}
$$

where $\mu_{q}=\frac{\mu}{\mu-(q+1)}, \mu \in\left(q+1, p_{s}^{*}\right)$. Thus $\mathcal{J}_{\lambda, M}$ is coercive and bounded below on $\mathcal{N}_{\lambda, M}(\Omega)$.

Lemma 2.2 Let (H1)-(H3) hold. There exists $\lambda_{1}>0$ such that for any $\lambda \in\left(0, \lambda_{1}\right)$, we have $\mathcal{N}_{\lambda, M}^{0}(\Omega)=\emptyset$.

Proof If not, that is, $\mathcal{N}_{\lambda, M}^{0}(\Omega) \neq \emptyset$ for each $\lambda>0$, then by (2.3) and the Hölder and Sobolev inequalities, we have for $u_{0} \in \mathcal{N}_{\lambda, M}^{0}(\Omega)$

$$
\begin{aligned}
a(r-p+1)\left\|u_{0}\right\|_{X_{0}}^{p} & \leq a(r-p+1)\left\|u_{0}\right\|_{X_{0}}^{p}+b\left(r-p^{2}+1\right)\left\|u_{0}\right\|_{X_{0}}^{p^{2}} \\
& =\lambda(r-q) \int_{\Omega} f\left|u_{0}\right|^{q+1} d x
\end{aligned}
$$

which implies that

$$
\begin{aligned}
\left\|u_{0}\right\|_{X_{0}}^{p} & \leq \frac{\lambda(r-q)}{a(r-p+1)} \int_{\Omega} f\left|u_{0}\right|^{q+1} d x \\
& \leq \frac{\lambda(r-q)}{a(r-p+1)}\|f\|_{L^{\mu_{q}}} S_{\mu}^{q+1}\left\|u_{0}\right\|_{X_{0}}^{q+1}
\end{aligned}
$$

and so

$$
\left\|u_{0}\right\|_{X_{0}} \leq\left(\frac{\lambda(r-q)}{a(r-p+1)}\|f\|_{L^{\mu q}} S_{\mu}^{q+1}\right)^{\frac{1}{p-q-1}} .
$$

Similarly, we obtain by (2.4) and the Hölder and Sobolev inequalities

$$
\left\|u_{0}\right\|_{X_{0}}^{p} \leq \frac{r-q}{a(p-q+1)}\|g\|_{L^{v_{r}}} S_{v}^{r+1}\left\|u_{0}\right\|_{X_{0}}^{r+1}
$$

which implies that

$$
\left\|u_{0}\right\|_{X_{0}} \geq\left(\frac{a(p-q+1)}{r-q}\|g\|_{L^{v_{r}}}^{-1} S_{v}^{-(r+1)}\right)^{\frac{1}{r-p+1}}
$$

But (2.5) contradicts (2.6) if $\lambda$ is sufficiently small. Hence, we conclude that there exists $\lambda_{1}>0$ such that $\mathcal{N}_{\lambda, M}^{0}(\Omega)=\emptyset$ for $\lambda \in\left(0, \lambda_{1}\right)$.

Let

$$
c_{\lambda}=\inf _{u \in \mathcal{N}_{\lambda, M}(\Omega)} \mathcal{J}_{\lambda, M}(u)
$$


From Lemma 2.2, for $\lambda \in\left(0, \lambda_{1}\right)$, we write $\mathcal{N}_{\lambda, M}(\Omega)=\mathcal{N}_{\lambda, M}^{+}(\Omega) \cup \mathcal{N}_{\lambda, M}^{-}(\Omega)$ and define

$$
c_{\lambda}^{+}=\inf _{u \in \mathcal{N}_{\lambda, M}^{+}(\Omega)} \mathcal{J}_{\lambda, M}(u) \quad \text { and } \quad c_{\lambda}^{-}=\inf _{u \in \mathcal{N}_{\lambda, M}^{-}(\Omega)} \mathcal{J}_{\lambda, M}(u)
$$

Lemma 2.3 (i) If $u \in \mathcal{N}_{\lambda, M}^{+}(\Omega)$, then $\int_{\Omega} f|u|^{q+1} d x>0$.

(ii) If $u \in \mathcal{N}_{\lambda, M}^{-}(\Omega)$, then $\int_{\Omega} g|u|^{r+1} d x>0$.

The proof is immediate from (2.3) and (2.4).

Define the function $k_{u}: \mathbb{R}^{+} \rightarrow \mathbb{R}$ as follows:

$$
k_{u}(t)=t^{p-q-1} M\left(t^{p}\|u\|_{X_{0}}^{p}\right)\|u\|_{X_{0}}^{p}-t^{r-q} \int_{\Omega} g|u|^{r+1} d x \quad t>0 .
$$

Obviously, $t u \in \mathcal{N}_{\lambda, M}(\Omega)$ if and only if $k_{u}(t)=\lambda \int_{\Omega} f|u|^{q+1} d x$. Moreover,

$$
\begin{aligned}
k_{u}^{\prime}(t)= & (p-q-1) t^{p-q-2} M\left(t^{p}\|u\|_{X_{0}}^{p}\right)\|u\|_{X_{0}}^{p}+p t^{2 p-q-2} M^{\prime}\left(t^{p}\|u\|_{X_{0}}^{p}\right)\|u\|_{X_{0}}^{2 p} \\
& -(r-q) t^{r-q-1} \int_{\Omega} g|u|^{r+1} d x
\end{aligned}
$$

which implies that $t^{q} k_{u}^{\prime}(t)=h_{\lambda, M}^{\prime \prime}(t)$ for $t u \in \mathcal{N}_{\lambda, M}(\Omega)$. That is, $u \in \mathcal{N}_{\lambda, M}^{+}(\Omega)$ (or $\left.\mathcal{N}_{\lambda, M}^{-}(\Omega)\right)$ if and only if $k_{u}^{\prime}(t)>0$ (or $\left.<0\right)$.

Set

$$
\begin{aligned}
A= & \frac{a(r-p+1)}{r-q}\left(\frac{a(p-q-1)}{(r-q)\|g\|_{L^{v_{r}} S_{v}^{r+1}}}\right)^{\frac{p-q-1}{r-p+1}} \\
& +\frac{b\left(r-p^{2}+1\right)}{r-q}\left(\frac{a(p-q-1)}{(r-q)\|g\|_{L^{v_{r}}} S_{v}^{r+1}}\right)^{\frac{p^{2}-q-1}{r-p+1}} .
\end{aligned}
$$

Lemma 2.4 Assume that (H1)-(H3) hold. Let $\lambda_{2}=\frac{A}{\|f\|_{L^{\mu q}} S_{\mu}^{q+1}}$. Then, for each $u \in X_{0} \backslash\{0\}$ and $\lambda \in\left(0, \lambda_{2}\right)$, we have:

(1) If $\int_{\Omega} f|u|^{q+1} d x \leq 0$, then there exists a unique $t^{-}=t^{-}(u)>t_{\max }(u)$ such that $t^{-} u \in \mathcal{N}_{\lambda, M}^{-}(\Omega)$ and

$$
\mathcal{J}_{\lambda, M}\left(t^{-} u\right)=\sup _{t \geq 0} \mathcal{J}_{\lambda, M}(t u)>0
$$

(2) If $\int_{\Omega} f|u|^{q+1} d x>0$, then there exists a unique $0<t^{+}=t^{+}(u)<t_{\max }(u)<t^{-}$such that $t^{+} u \in \mathcal{N}_{\lambda, M}^{+}(\Omega), t^{-} u \in \mathcal{N}_{\lambda, M}^{-}(\Omega)$ and

$$
\mathcal{J}_{\lambda, M}\left(t^{+} u\right)=\inf _{0 \leq t \leq t_{\max }(u)} \mathcal{J}_{\lambda, M}(t u), \quad \mathcal{J}_{\lambda, M}\left(t^{-} u\right)=\sup _{t \geq 0} \mathcal{J}_{\lambda, M}(t u)
$$

Proof From (2.7) and (2.8), we have

$$
k_{u}(t)=a t^{p-q-1}\|u\|_{X_{0}}^{p}+b t^{p^{2}-q-1}\|u\|_{X_{0}}^{p^{2}}-t^{r-q} \int_{\Omega} g|u|^{r+1} d x \quad t \geq 0,
$$

and

$$
k_{u}^{\prime}(t)=t^{-q-1}\left[a(p-q-1) t^{p-1}\|u\|_{X_{0}}^{p}+b\left(p^{2}-q-1\right) t^{p^{2}-1}\|u\|_{X_{0}}^{p^{2}}-(r-q) t^{r} \int_{\Omega} g|u|^{r+1} d x\right]
$$


which implies that $k_{u}(0)=0, k_{u}(t) \rightarrow-\infty$ as $t \rightarrow \infty, \lim _{t \rightarrow 0^{+}} k_{u}^{\prime}(t)>0$ and $\lim _{t \rightarrow \infty} k_{u}^{\prime}(t)<0$. Thus there exists a unique $t_{\max }(u):=t_{\max }>0$ such that $k_{u}(t)$ is increasing on $\left(0, t_{\max }\right)$, decreasing on $\left(t_{\max }, \infty\right)$ and $k_{u}^{\prime}\left(t_{\max }\right)=0$. Moreover, $t_{\max }$ is the root of

$$
a(p-q-1) t_{\max }^{p-1}\|u\|_{X_{0}}^{p}+b\left(p^{2}-q-1\right) t_{\max }^{p^{2}-1}\|u\|_{X_{0}}^{p^{2}}-(r-q) t_{\max }^{r} \int_{\Omega} g|u|^{r+1} d x=0 .
$$

From (2.12), we obtain

$$
t_{\max } \geq\left(\frac{a(p-q-1)\|u\|_{X_{0}}^{p}}{(r-q) \int_{\Omega} g|u|^{r+1} d x}\right)^{\frac{1}{r-p+1}} \geq \frac{1}{\|u\|_{X_{0}}}\left(\frac{a(p-q-1)}{(r-q)\|g\|_{L^{v r}} S_{v}^{r+1}}\right)^{\frac{1}{r-p+1}}:=t_{*} .
$$

Hence, we have by (2.12), (2.13), and the Hölder and Sobolev inequalities

$$
\begin{aligned}
k_{u}\left(t_{\max }\right)= & t_{\max }^{p-q-1}\left[a\|u\|_{X_{0}}^{p}+b t_{\max }^{p(p-1)}\|u\|_{X_{0}}^{p^{2}}-t_{\max }^{r-p+1} \int_{\Omega} g|u|^{r+1} d x\right] \\
= & \frac{a(r-p+1)}{r-q} t_{\max }^{p-q-1}\|u\|_{X_{0}}^{p}+\frac{b\left(r-p^{2}+1\right)}{r-q} t_{\max }^{p^{2}-q-1}\|u\|_{X_{0}}^{p^{2}} \\
\geq & \frac{a(r-p+1)}{r-q} t_{*}^{p-q-1}\|u\|_{X_{0}}^{p}+\frac{b\left(r-p^{2}+1\right)}{r-q} t_{*}^{p^{2}-q-1}\|u\|_{X_{0}}^{p^{2}} \\
\geq & \frac{a(r-p+1)}{r-q}\left(\frac{a(p-q-1)}{(r-q)\|g\|_{L^{v r}} S_{v}^{r+1}}\right)^{\frac{p-q-1}{r-p+1}}\|u\|_{X_{0}}^{q+1} \\
& +\frac{b\left(r-p^{2}+1\right)}{r-q}\left(\frac{a(p-q-1)}{(r-q)\|g\|_{L^{v r}} S_{v}^{r+1}}\right)^{\frac{p^{2}-q-1}{r-p+1}}\|u\|_{X_{0}}^{q+1} \\
= & A\|u\|_{X_{0}}^{q+1} .
\end{aligned}
$$

Case (1): $\int_{\Omega} f|u|^{q+1} d x \leq 0$. Then $k_{u}(t)=\lambda \int_{\Omega} f|u|^{q+1} d x$ has unique solution $t^{-}>t_{\max }$ and $k_{u}^{\prime}\left(t^{-}\right)<0$. On the other hand, we have

$$
\begin{aligned}
a(p-q-1) & \left\|t^{-} u\right\|_{X_{0}}^{p}+b\left(p^{2}-q-1\right)\left\|t^{-} u\right\|_{X_{0}}^{p^{2}}-(r-q) \int_{\Omega} g\left|t^{-} u\right|^{r+1} d x \\
= & \left(t^{-}\right)^{2+q}\left[a(p-q-1)\left(t^{-}\right)^{p-q-2}\|u\|_{X_{0}}^{p}+b\left(p^{2}-q-1\right)\left(t^{-}\right)^{p^{2}-q-2}\|u\|_{X_{0}}^{p^{2}}\right. \\
& \left.\quad-(r-q)\left(t^{-}\right)^{r-q-1} \int_{\Omega} g|u|^{r+1} d x\right] \\
= & \left(t^{-}\right)^{2+q} k_{u}^{\prime}\left(t^{-}\right)<0
\end{aligned}
$$

and

$$
\begin{aligned}
& \left\langle\mathcal{J}_{\lambda, M}^{\prime}\left(t^{-} u\right), t^{-} u\right\rangle \\
& \quad=a\left(t^{-}\right)^{p}\|u\|_{X_{0}}^{p}+b\left(t^{-}\right)^{p^{2}}\|u\|_{X_{0}}^{p^{2}}-\lambda\left(t^{-}\right)^{q+1} \int_{\Omega} f|u|^{q+1} d x-\left(t^{-}\right)^{r+1} \int_{\Omega} g|u|^{r+1} d x \\
& \quad=\left(t^{-}\right)^{q+1}\left[k_{u}\left(t^{-}\right)-\lambda \int_{\Omega} f|u|^{q+1} d x\right]=0 .
\end{aligned}
$$


Hence, $t^{-} u \in \mathcal{N}_{\lambda, M}^{-}(\Omega)$ or $t^{-}=1$. For $t>t_{\max }$, we obtain

$$
\begin{aligned}
& a(p-q-1)\|t u\|_{X_{0}}^{p}+b\left(p^{2}-q-1\right)\|t u\|_{X_{0}}^{p^{2}}-(r-q) \int_{\Omega} g|t u|^{r+1} d x<0, \\
& \frac{d^{2}}{d t^{2}} \mathcal{J}_{\lambda, M}(t u)<0, \\
& \frac{d}{d t} \mathcal{J}_{\lambda, M}(t u)=a t^{p-1}\|u\|_{X_{0}}^{p}+b t^{p^{2}-1}\|u\|_{X_{0}}^{p^{2}}-\lambda t^{q} \int_{\Omega} f|u|^{q+1} d x-t^{r} \int_{\Omega} g|u|^{r+1} d x=0,
\end{aligned}
$$

for $t=t^{-}$. Thus, $\mathcal{J}_{\lambda, M}(u)=\sup _{t \geq 0} \mathcal{J}_{\lambda, M}(t u)$. Furthermore, we have

$$
\mathcal{J}_{\lambda, M}(u) \geq \mathcal{J}_{\lambda, M}(t u) \geq \frac{a}{p} t^{p}\|u\|_{X_{0}}^{p}+\frac{b}{p^{2}} t^{p^{2}}\|u\|_{X_{0}}^{p^{2}}-\frac{1}{r+1} t^{r+1} \int_{\Omega} g|u|^{r+1} d x, \quad t \geq 0 .
$$

Let

$$
h_{u}(t)=\frac{a}{p} t^{p}\|u\|_{X_{0}}^{p}+\frac{b}{p^{2}} t^{p^{2}}\|u\|_{X_{0}}^{p^{2}}-\frac{1}{r+1} t^{r+1} \int_{\Omega} g|u|^{r+1} d x, \quad t \geq 0 .
$$

Similar to the argument in the function $k_{u}(t)$, we see that $h_{u}(t)$ achieves its maximum at $t_{m} \geq\left(\frac{a\|u\|_{X_{0}}^{p}}{\int_{\Omega} g|u|^{r+1} d x}\right)^{\frac{1}{r-p+1}}$. Thus, we have

$$
\mathcal{J}_{\lambda, M}(u) \geq h_{u}\left(t_{m}\right) \geq \frac{a p(r+1-p)+b\left(r+1-p^{2}\right)}{p^{2}(r+1)}\left(\frac{a\|u\|_{X_{0}}^{r+1}}{\int_{\Omega} g|u|^{r+1} d x}\right)^{\frac{p}{r-p+1}}>0
$$

Case (2): $\int_{\Omega} f|u|^{q+1} d x>0$. By (2.14) and

$$
\begin{aligned}
k_{u}(0) & =0<\lambda \int_{\Omega} f|u|^{q+1} d x \leq \lambda\|f\|_{L^{\mu q}}^{\mu_{\mu}} S_{\mu}^{q+1}\|u\|_{X_{0}}^{q+1} \\
& <\lambda_{2}\|f\|_{L^{\mu_{q}}} S_{\mu}^{q+1}\|u\|_{X_{0}}^{q+1}=A\|u\|_{X_{0}}^{q+1} \leq k_{u}\left(t_{\max }\right), \quad \text { for } \lambda \in\left(0, \lambda_{2}\right) .
\end{aligned}
$$

Then there exist $t^{+}$and $t^{-}$such that $0<t^{+}<t_{\max }<t^{-}$,

$$
k_{u}\left(t^{+}\right)=\lambda \int_{\Omega} f|u|^{q+1} d x=k_{u}\left(t^{-}\right)
$$

Moreover, we have $k_{u}^{\prime}\left(t^{+}\right)>0$ and $k_{u}^{\prime}\left(t^{-}\right)<0$. Thus, there are two multiples of $u$ lying in $\mathcal{N}_{\lambda, M}(\Omega)$, that is, $t^{+} u \in \mathcal{N}_{\lambda, M}^{+}(\Omega)$ and $t^{-} u \in \mathcal{N}_{\lambda, M}^{-}(\Omega)$, and $\mathcal{J}_{\lambda, M}\left(t^{-} u\right) \geq \mathcal{J}_{\lambda, M}(t u) \geq \mathcal{J}_{\lambda, M}\left(t^{+} u\right)$ for each $t \in\left[t^{+}, t^{-}\right]$and $\mathcal{J}_{\lambda, M}\left(t^{+} u\right) \leq \mathcal{J}_{\lambda, M}(t u)$ for each $t \in\left[0, t^{+}\right]$. Hence, $t^{-}=1$ and

$$
\mathcal{J}_{\lambda, M}(u)=\sup _{t \geq 0} \mathcal{J}_{\lambda, M}(t u), \quad \mathcal{J}_{\lambda, M}\left(t^{+} u\right)=\inf _{0 \leq t \leq t_{\max }} \mathcal{J}_{\lambda, M}(t u) .
$$

Lemma 2.5 If $(\mathrm{H} 3)$ holds, then we have $c_{\lambda} \leq c_{\lambda}^{+}<0$.

Proof For $u \in \mathcal{N}_{\lambda, M}^{+}$, we get

$$
(r-q) \lambda \int_{\Omega} f|u|^{q+1} d x>a(r-p+1)\|u\|_{X_{0}}^{p}+b\left(r-p^{2}+1\right)\|u\|_{X_{0}}^{p^{2}}
$$


Thus, we have

$$
\begin{aligned}
J_{\lambda, M}(u) & =\frac{a(r-p+1)}{p(r+1)}\|u\|_{X_{0}}^{p}+\frac{b\left(r-p^{2}+1\right)}{p^{2}(r+1)}\|u\|_{X_{0}}^{p^{2}}-\frac{\lambda(r-q)}{(q+1)(r+1)} \int_{\Omega} f|u|^{q+1} d x \\
& <\frac{a(r-p+1)}{r+1}\left[\frac{1}{p}-\frac{1}{q+1}\right]\|u\|_{X_{0}}^{p}+\frac{b\left(r-p^{2}+1\right)}{r+1}\left[\frac{1}{p^{2}}-\frac{1}{q+1}\right]\|u\|_{X_{0}}^{p^{2}}<0,
\end{aligned}
$$

which implies that $c_{\lambda} \leq c_{\lambda}^{+}<0$.

\section{Main results}

Using the idea of Ni-Takagi [10], we have the following.

Lemma 3.1 For each $u \in \mathcal{N}_{\lambda, M}(\Omega)$, there exist $\epsilon>0$ and a differentiable function $\xi$ : $B(0 ; \epsilon) \subset X_{0} \rightarrow \mathbb{R}^{+}$such that $\xi(0)=1$, the function $\xi(v)(u-v) \in \mathcal{N}_{\lambda, M}(\Omega)$ and

$$
\left\langle\xi^{\prime}(0), v\right\rangle=\frac{W}{a(p-q-1)\|u\|_{X_{0}}^{p}+b\left(p^{2}-q-1\right)\|u\|_{X_{0}}^{p^{2}}-(r-q) \int_{\Omega} g|u|^{r+1} d x}
$$

for all $v \in X_{0}$, where

$$
\begin{aligned}
W= & a p \int_{Q} \frac{|u(x)-u(y)|^{p-2}(u(x)-u(y))(v(x)-v(y))}{|x-y|^{N+s p}} d x d y \\
& +b p^{2} \int_{Q} \frac{|u(x)-u(y)|^{p^{2}-2}(u(x)-u(y))(v(x)-v(y))}{|x-y|^{N+s p^{2}}} d x d y \\
& -(q+1) \lambda \int_{\Omega} f|u|^{q-1} u v d x-(r+1) \int_{\Omega} g|u|^{r-1} u v d x .
\end{aligned}
$$

Proof For $u \in \mathcal{N}_{\lambda, M}(\Omega)$, we define a function $\mathcal{F}: \mathbb{R} \times X_{0} \rightarrow \mathbb{R}$ by

$$
\begin{aligned}
\mathcal{F}_{u}(\xi, w)= & \left\langle\mathcal{J}_{\lambda, M}^{\prime}(\xi(u-w)), \xi(u-w)\right\rangle \\
= & \xi^{p} M\left(\xi^{p}\|u-w\|_{X_{0}}^{p}\right)\|u-w\|_{X_{0}}^{p} \\
& -\xi^{q+1} \lambda \int_{\Omega} f|u-w|^{q+1} d x-\xi^{r+1} \int_{\Omega} g|u-w|^{r+1} d x \\
= & a \xi^{p}\|u-w\|_{X_{0}}^{p}+b \xi^{p^{2}}\|u-w\|_{X_{0}}^{p^{2}} \\
& -\xi^{q+1} \lambda \int_{\Omega} f|u-w|^{q+1} d x-\xi^{r+1} \int_{\Omega} g|u-w|^{r+1} d x .
\end{aligned}
$$

Then $\mathcal{F}_{u}(1,0)=\left\langle\mathcal{J}_{\lambda, M}^{\prime}(u), u\right\rangle=0$ and

$$
\begin{aligned}
\frac{d}{d \xi} \mathcal{F}_{u}(1,0) & =a p\|u\|_{X_{0}}^{p}+b p^{2}\|u\|_{X_{0}}^{p^{2}}-(q+1) \lambda \int_{\Omega} f|u|^{q+1} d x-(r+1) \int_{\Omega} g|u|^{r+1} d x \\
& =a(p-q-1)\|u\|_{X_{0}}^{p}+b\left(p^{2}-q-1\right)\|u\|_{X_{0}}^{p^{2}}-(r-q) \int_{\Omega} g|u|^{r+1} d x \neq 0 .
\end{aligned}
$$


From the implicit function theorem, we know that there exist $\epsilon>0$ and a differentiable function $\xi: B(0 ; \epsilon) \subset X_{0} \rightarrow \mathbb{R}$ such that $\xi(0)=1$,

$$
\left\langle\xi^{\prime}(0), v\right\rangle=\frac{W}{a(p-q-1)\|u\|_{X_{0}}^{p}+b\left(p^{2}-q-1\right)\|u\|_{X_{0}}^{p^{2}}-(r-q) \int_{\Omega} g|u|^{r+1} d x},
$$

where $W$ is as in (3.2), and

$$
\mathcal{F}_{u}(\xi(v), v)=0 \quad \text { for all } v \in B(0 ; \epsilon)
$$

which is equivalent to

$$
\left\langle\mathcal{J}_{\lambda, M}^{\prime}(\xi(v)(u-v)), \xi(v)(u-v)\right\rangle=0 \quad \text { for all } v \in B(0 ; \epsilon)
$$

which implies that $\xi(v)(u-v) \in \mathcal{N}_{\lambda, M}(\Omega)$.

Similar to the argument in Lemma 3.1, we can obtain the following lemma.

Lemma 3.2 For each $u \in \mathcal{N}_{\lambda, M}^{-}(\Omega)$, there exist $\epsilon>0$ and a differentiable function $\xi^{-}$: $B(0 ; \epsilon) \subset X_{0} \rightarrow \mathbb{R}^{+}$such that $\xi^{-}(0)=1$, the function $\xi^{-}(v)(u-v) \in \mathcal{N}_{\lambda, M}^{-}(\Omega)$ and

$$
\left\langle\left(\xi^{-}\right)^{\prime}(0), v\right\rangle=\frac{W}{a(p-q-1)\|u\|_{X_{0}}^{p}+b\left(p^{2}-q-1\right)\|u\|_{X_{0}}^{p^{2}}-(r-q) \int_{\Omega} g|u|^{r+1} d x},
$$

for all $v \in X_{0}$, where $W$ is as in (3.2).

Let

(H4) $p<2+\frac{(r-1) q}{r}$.

Moreover, we let

$$
p^{*}=\frac{(p-2) r}{r-1}-q
$$

and

$$
\begin{aligned}
\lambda_{3}= & \left(\frac{a(p-q-1)\left(r-p^{2}+1\right)}{(r-q)\left(p^{2}-q-1\right)}\right)\left(\frac{a(p-q-1)}{r-q}\right)^{\frac{(p-q-1)}{\left(p-q-1-p^{*}\right)(r-1)}} \\
& \times\left(\frac{1}{\|f\|_{L^{\mu q}} S_{\mu}^{q+1}}\right)\left(\frac{1}{\|g\|_{L^{v_{r}}} S_{\nu}^{r+1}}\right)^{\frac{(p-q-1)}{(r-1)\left(p-q-1-p^{*}\right)}} .
\end{aligned}
$$

Remark 3.1 By (H4) we know that $p^{*}<0$.

Lemma 3.3 Assume that (H1)-(H4) hold. Let $\Gamma_{0}=\min \left\{\lambda_{1}, \lambda_{2}, \lambda_{3}\right\}$, then for $\lambda \in\left(0, \Gamma_{0}\right)$ :

(i) There exists a minimizing sequence $\left\{u_{n}\right\} \subset \mathcal{N}_{\lambda, M}(\Omega)$ such that

$$
\mathcal{J}_{\lambda, M}\left(u_{n}\right)=c_{\lambda}+o(1), \quad \mathcal{J}_{\lambda, M}^{\prime}\left(u_{n}\right)=o(1) \quad \text { in }\left(X_{0}\right)^{*} .
$$


(ii) There exists a minimizing sequence $\left\{u_{n}\right\} \subset \mathcal{N}_{\lambda, M}^{-}(\Omega)$ such that

$$
\mathcal{J}_{\lambda, M}\left(u_{n}\right)=c_{\lambda}^{-}+o(1), \quad \mathcal{J}_{\lambda, M}^{\prime}\left(u_{n}\right)=o(1) \quad \text { in }\left(X_{0}\right)^{*}
$$

Proof By the Ekeland variational principle [11] and Lemma 2.2, there exists a minimizing sequence $\left\{u_{n}\right\} \subset \mathcal{N}_{\lambda, M}(\Omega)$ such that

$$
\mathcal{J}_{\lambda, M}\left(u_{n}\right)<c_{\lambda}+\frac{1}{n}
$$

and

$$
\mathcal{J}_{\lambda, M}\left(u_{n}\right)<\mathcal{J}_{\lambda, M}(w)+\frac{1}{n}\left\|w-u_{n}\right\|_{X_{0}} \quad \forall w \in \mathcal{N}_{\lambda, M}(\Omega)
$$

Let $n$ large enough, by Lemma 2.5, we obtain

$$
\begin{aligned}
\mathcal{J}_{\lambda, M}\left(u_{n}\right) & =\frac{a(r-p+1)}{p(r+1)}\left\|u_{n}\right\|_{X_{0}}^{p}+\frac{b\left(r-p^{2}+1\right)}{p^{2}(r+1)}\left\|u_{n}\right\|_{X_{0}}^{p^{2}}-\frac{\lambda(r-q)}{(q+1)(r+1)} \int_{\Omega} f\left|u_{n}\right|^{q+1} d x \\
& <c_{\lambda}+\frac{1}{n}<\frac{c_{\lambda}}{2},
\end{aligned}
$$

which implies that

$$
\|f\|_{L^{\mu_{q}}} S_{\mu}^{q+1}\left\|u_{n}\right\|_{X_{0}}^{q+1} \geq \int_{\Omega} f\left|u_{n}\right|^{q+1} d x>-\frac{(q+1)(r+1)}{\lambda(r-q)} \frac{c_{\lambda}}{2}>0 .
$$

This implies $u_{n} \neq 0$ and by using (3.4), (3.5), and the Hölder inequality, we get

$$
\left\|u_{n}\right\|_{X_{0}}>\left[-\frac{(q+1)(r+1)}{\lambda(r-q)} \frac{c_{\lambda}}{2}\|f\|_{L^{\mu q}}^{-1} S_{\mu}^{-(q+1)}\right]^{\frac{1}{q+1}}
$$

and

$$
\left\|u_{n}\right\|_{X_{0}}<\left[\frac{\lambda p(r-q)(r+1)}{a(q+1)(r+1)(r-p+1)}\|f\|_{L^{\mu_{q}}} S_{\mu}^{q+1}\right]^{\frac{1}{p-q-1}} .
$$

In the following, we will prove that

$$
\left\|\mathcal{J}_{\lambda, M}^{\prime}\left(u_{n}\right)\right\|_{\left(X_{0}\right)^{*}} \rightarrow 0 \quad \text { as } n \rightarrow \infty
$$

By using Lemma 3.1 with $u_{n}$ we get the functions $\xi_{n}: B\left(0 ; \epsilon_{n}\right) \rightarrow \mathbb{R}^{+}$for some $\epsilon_{n}>0$, such that $\xi_{n}(w)\left(u_{n}-w\right) \in \mathcal{N}_{\lambda, M}(\Omega)$. For fixed $n \in \mathbb{N}$, we choose $0<\rho<\epsilon_{n}$. Let $u \in X_{0}$ with $u \neq 0$ and let $w_{\rho}=\frac{\rho u}{\|u\|_{X_{0}}}$. Set $\eta_{\rho}=\xi_{n}\left(w_{\rho}\right)\left(u_{n}-w_{\rho}\right)$, since $\eta_{\rho} \in \mathcal{N}_{\lambda, M}(\Omega)$, we deduce from (3.4) that

$$
\mathcal{J}_{\lambda, M}\left(\eta_{\rho}\right)-J_{\lambda, M}\left(u_{n}\right) \geq-\frac{1}{n}\left\|\eta_{\rho}-u_{n}\right\|_{X_{0}} \quad \forall w \in \mathcal{N}_{\lambda, M}(\Omega)
$$

and by the mean value theorem, we obtain

$$
\left\langle\mathcal{J}_{\lambda, M}^{\prime}\left(u_{n}\right), \eta_{\rho}-u_{n}\right\rangle+o\left(\left\|\eta_{\rho}-u_{n}\right\|_{X_{0}}\right) \geq-\frac{1}{n}\left\|\eta_{\rho}-u_{n}\right\|_{X_{0}} .
$$


Hence,

$$
\begin{aligned}
& \left\langle\mathcal{J}_{\lambda, M}^{\prime}\left(u_{n}\right),-w_{\rho}\right\rangle+\left(\xi_{n}\left(w_{\rho}\right)-1\right)\left\langle\mathcal{J}_{\lambda, M}^{\prime}\left(u_{n}\right), u_{n}-w_{\rho}\right\rangle \\
& \geq-\frac{1}{n}\left\|\eta_{\rho}-u_{n}\right\|_{X_{0}}+o\left(\left\|\eta_{\rho}-u_{n}\right\|_{X_{0}}\right) .
\end{aligned}
$$

By $\xi_{n}\left(w_{\rho}\right)\left(u_{n}-w_{\rho}\right) \in \mathcal{N}_{\lambda, M}(\Omega)$ and (3.8) it follows that

$$
\begin{aligned}
& -\rho\left\langle\mathcal{J}_{\lambda, M}^{\prime}\left(u_{n}\right), \frac{u}{\|u\|_{X_{0}}}\right\rangle+\left(\xi_{n}\left(w_{\rho}\right)-1\right)\left\langle\mathcal{J}_{\lambda, M}^{\prime}\left(u_{n}\right)-\mathcal{J}_{\lambda, M}^{\prime}\left(\eta_{\rho}\right), u_{n}-w_{\rho}\right\rangle \\
& \geq-\frac{1}{n}\left\|\eta_{\rho}-u_{n}\right\|_{X_{0}}+o\left(\left\|\eta_{\rho}-u_{n}\right\|_{X_{0}}\right) .
\end{aligned}
$$

Thus,

$$
\begin{aligned}
\left\langle\mathcal{J}_{\lambda, M}^{\prime}\left(u_{n}\right), \frac{u}{\|u\|_{X_{0}}}\right\rangle \leq & \frac{1}{n \rho}\left\|\eta_{\rho}-u_{n}\right\|_{X_{0}}+\frac{1}{\rho} o\left(\left\|\eta_{\rho}-u_{n}\right\|_{X_{0}}\right) \\
& +\frac{\left(\xi_{n}\left(w_{\rho}\right)-1\right)}{\rho}\left\langle\mathcal{J}_{\lambda, M}^{\prime}\left(u_{n}\right)-\mathcal{J}_{\lambda, M}^{\prime}\left(\eta_{\rho}\right), u_{n}-w_{\rho}\right\rangle .
\end{aligned}
$$

Since

$$
\left\|\eta_{\rho}-u_{n}\right\|_{X_{0}} \leq \rho\left|\xi_{n}\left(w_{\rho}\right)\right|+\left|\xi_{n}\left(w_{\rho}\right)-1\right|\left\|u_{n}\right\|_{X_{0}}
$$

and

$$
\lim _{n \rightarrow \infty} \frac{\left|\xi_{n}\left(w_{\rho}\right)-1\right|}{\rho} \leq\left\|\xi_{n}^{\prime}(0)\right\|
$$

taking the limit $\rho \rightarrow 0$ in (3.9), we obtain

$$
\left\langle\mathcal{J}_{\lambda, M}^{\prime}\left(u_{n}\right), \frac{u}{\|u\|_{X_{0}}}\right\rangle \leq \frac{C}{n}\left(1+\left\|\xi_{n}^{\prime}(0)\right\|\right)
$$

for some constant $C>0$, independent of $\rho$. In the following, we will show that $\left\|\xi_{n}^{\prime}(0)\right\|$ is uniformly bounded in $n$. From (3.1), (3.7), and the Hölder inequality, we obtain for some $\kappa>0$

$$
\left\langle\xi_{n}^{\prime}(0), v\right\rangle \leq \frac{\kappa\|v\|_{X_{0}}}{a(p-q-1)\left\|u_{n}\right\|_{X_{0}}^{p}+b\left(p^{2}-q-1\right)\left\|u_{n}\right\|_{X_{0}}^{p^{2}}-(r-q) \int_{\Omega} g\left|u_{n}\right|^{r+1} d x} .
$$

We only need to prove that

$$
\left.\left|a(p-q-1)\left\|u_{n}\right\|_{X_{0}}^{p}+b\left(p^{2}-q-1\right)\left\|u_{n}\right\|_{X_{0}}^{p^{2}}-(r-q) \int_{\Omega} g\right| u_{n}\right|^{r+1} d x \mid>c
$$

for some $c>0$ and $n$ large enough. If (3.10) is fails, then there exists a subsequence $\left\{u_{n}\right\}$ such that

$$
a(p-q-1)\left\|u_{n}\right\|_{X_{0}}^{p}+b\left(p^{2}-q-1\right)\left\|u_{n}\right\|_{X_{0}}^{p^{2}}-(r-q) \int_{\Omega} g\left|u_{n}\right|^{r+1} d x=o(1) .
$$


Combining (3.11) with (3.6), we may find a suitable constant $d>0$ such that

$$
\int_{\Omega} g\left|u_{n}\right|^{r+1} d x \geq d \quad \text { for } n \text { sufficiently large. }
$$

By (3.11) and $u_{n} \in \mathcal{N}_{\lambda, M}(\Omega)$, we have

$$
\begin{aligned}
& \lambda \int_{\Omega} f\left|u_{n}\right|^{q+1} d x \\
& \quad=a\left\|u_{n}\right\|_{X_{0}}^{p}+b\left\|u_{n}\right\|_{X_{0}}^{p^{2}}-\int_{\Omega} g\left|u_{n}\right|^{r+1} d x \\
& \quad=\frac{1}{p^{2}-q-1}\left(a\left(p^{2}-q-1\right)\left\|u_{n}\right\|_{X_{0}}^{p}+b\left(p^{2}-q-1\right)\left\|u_{n}\right\|_{X_{0}}^{p^{2}}\right)-\int_{\Omega} g\left|u_{n}\right|^{r+1} d x \\
& \quad \geq \frac{1}{p^{2}-q-1}\left(a(p-q-1)\left\|u_{n}\right\|_{X_{0}}^{p}+b\left(p^{2}-q-1\right)\left\|u_{n}\right\|_{X_{0}}^{p^{2}}\right)-\int_{\Omega} g\left|u_{n}\right|^{r+1} d x \\
& \quad=\frac{r-q}{p^{2}-q-1} \int_{\Omega} g\left|u_{n}\right|^{r+1} d x-\int_{\Omega} g\left|u_{n}\right|^{r+1} d x+o(1) \\
& \quad=\frac{r-p^{2}+1}{p^{2}-q-1} \int_{\Omega} g\left|u_{n}\right|^{r+1} d x+o(1) .
\end{aligned}
$$

Moreover, we have by (3.11) and (3.13)

$$
\begin{aligned}
a(p-q-1)\left\|u_{n}\right\|_{X_{0}}^{p} & \leq a(p-q-1)\left\|u_{n}\right\|_{X_{0}}^{p}+b\left(p^{2}-q-1\right)\left\|u_{n}\right\|_{X_{0}}^{p^{2}} \\
& =(r-q) \int_{\Omega} g\left|u_{n}\right|^{r+1} d x+o(1) \\
& \leq \lambda \frac{\left(p^{2}-q-1\right)(r-q)}{r-p^{2}+1} \int_{\Omega} f\left|u_{n}\right|^{q+1} d x+o(1) \\
& \leq \lambda \frac{\left(p^{2}-q-1\right)(r-q)}{r-p^{2}+1}\|f\|_{L^{\mu q}} S_{\mu}^{q+1}\left\|u_{n}\right\|_{X_{0}}^{q+1}+o(1),
\end{aligned}
$$

which implies that

$$
\left\|u_{n}\right\|_{X_{0}} \leq\left(\lambda \frac{\left(p^{2}-q-1\right)(r-q)}{a(p-q-1)\left(r-p^{2}+1\right)}\|f\|_{L^{\mu q}} S_{\mu}^{q+1}\right)^{\frac{1}{p-q-1}}+o(1) .
$$

Let

$$
\mathcal{I}_{\lambda, M}(u)=K(p, q, r)\left(\frac{\|u\|_{X_{0}}^{p r}}{\int_{\Omega} g\left|u_{n}\right|^{r+1} d x}\right)^{\frac{1}{r-1}}-\lambda \int_{\Omega} f|u|^{q+1} d x,
$$

where

$$
K(p, q, r)=\left(\frac{a(p-q-1)}{r-q}\right)^{\frac{r}{r-1}} \frac{r-p^{2}+1}{p^{2}-q-1} .
$$

From (3.11), it is easy to see that

$$
\left\|u_{n}\right\|_{X_{0}}^{p} \leq \frac{r-q}{a(p-q-1)} \int_{\Omega} g\left|u_{n}\right|^{r+1} d x .
$$


Thus,

$$
\begin{aligned}
\mathcal{I}_{\lambda, M}\left(u_{n}\right) \leq & \left(\frac{a(p-q-1)}{r-q}\right)^{\frac{r}{r-1}} \frac{r-p^{2}+1}{p^{2}-q-1}\left(\frac{\left(\frac{r-q}{a(p-q-1)}\right)^{r}\left(\int_{\Omega} g\left|u_{n}\right|^{r+1} d x\right)^{r}}{\int_{\Omega} g\left|u_{n}\right|^{r+1} d x}\right)^{\frac{1}{r-1}} \\
& -\frac{r-p^{2}+1}{p^{2}-q-1} \int_{\Omega} g\left|u_{n}\right|^{r+1} d x+o(1) \\
= & o(1) .
\end{aligned}
$$

But, by (3.12), (3.14), and $\lambda \in \Gamma_{0}$,

$$
\begin{aligned}
\mathcal{I}_{\lambda, M}\left(u_{n}\right) \geq & K(p, q, r)\left(\frac{\left\|u_{n}\right\|_{X_{0}}^{p r}}{\|g\|_{L^{v r}} S_{v}^{r+1}\left\|u_{n}\right\|_{X_{0}}^{r+1}}\right)^{\frac{1}{r-1}}-\lambda\|f\|_{L^{\mu}} S_{\mu}^{q+1}\left\|u_{n}\right\|_{X_{0}}^{q+1} \\
= & \left\|u_{n}\right\|_{X_{0}}^{q+1}\left(K(p, q, r)\|g\|_{L^{v_{r}}}^{\frac{1}{1-r}} S_{v}^{\frac{r+1}{1-r}}\left\|u_{n}\right\|_{X_{0}}^{p^{*}}-\lambda\|f\|_{L^{\mu}} S_{\mu}^{q+1}\right) \\
\geq & \left\|u_{n}\right\|_{X_{0}}^{q+1}\left\{K(p, q, r)\|g\|_{L^{v r}}^{\frac{1}{1-r}} S_{\nu}^{\frac{r+1}{1-r}}\left[\lambda \frac{\left(p^{2}-q-1\right)(r-q)}{a(p-q-1)\left(r-p^{2}+1\right)}\|f\|_{L^{\mu q}} S_{\mu}^{q+1}\right]^{\frac{p^{*}}{p-q-1}}\right. \\
& \left.-\lambda\|f\|_{L^{\mu q}} S_{\mu}^{q+1}\right\},
\end{aligned}
$$

which contradicts (3.16), where $p^{*}=\frac{(p-2) r}{r-1}-q<0$.

Hence, we obtain

$$
\left\langle\mathcal{J}_{\lambda, M}^{\prime}\left(u_{n}\right), \frac{u}{\|u\|_{X_{0}}}\right\rangle \leq \frac{C}{n} .
$$

This completes the proof of (i). Similarly, we can prove (ii) by using Lemma 3.2.

Theorem 3.4 Assume that (H1)-(H4) hold. For each $0<\lambda<\Gamma_{0}\left(\Gamma_{0}\right.$ is as in Lemma 3.3), the functional $\mathcal{J}_{\lambda, M}$ has a minimizer $u_{\lambda}^{+}$in $\mathcal{N}_{\lambda, M}^{+}(\Omega)$ satisfying:

(1) $\mathcal{J}_{\lambda, M}\left(u_{\lambda}^{+}\right)=c_{\lambda}^{+}=c_{\lambda}$;

(2) $u_{\lambda}^{+}$is a solution of (1.1).

Proof By Lemma 3.3(i), there exists a minimizing sequence $\left\{u_{n}\right\} \subset \mathcal{N}_{\lambda, M}(\Omega)$ for $\mathcal{J}_{\lambda, M}$ on $\mathcal{N}_{\lambda, M}(\Omega)$ such that

$$
\mathcal{J}_{\lambda, M}\left(u_{n}\right)=c_{\lambda}+o(1), \quad \mathcal{J}_{\lambda, M}^{\prime}\left(u_{n}\right)=o(1) \quad \text { in }\left(X_{0}\right)^{*}
$$

From Lemma 2.5 and the compact embedding theorem, we see that there exist a subsequence $\left\{u_{n}\right\}$ and $u_{\lambda}^{+} \in X_{0}$ such that

$$
u_{n} \rightarrow u_{\lambda}^{+} \quad \text { weakly in } X_{0}
$$

and

$$
u_{n} \rightarrow u_{\lambda}^{+} \quad \text { strongly in } L^{\eta}(\Omega) \text { for } 1<\eta<p_{s}^{*} .
$$


In the following we will prove that $\int_{\Omega} f\left|u_{\lambda}^{+}\right|^{q+1} d x \neq 0$. In fact, if not, by (3.17) and the Hölder inequality we can obtain

$$
\int_{\Omega} f\left|u_{n}\right|^{q+1} d x \rightarrow \int_{\Omega} f\left|u_{\lambda}^{+}\right|^{q+1} d x=0
$$

as $n \rightarrow \infty$. Hence,

$$
a\left\|u_{n}\right\|_{X_{0}}^{p}+b\left\|u_{n}\right\|_{X_{0}}^{p^{2}}=\int_{\Omega} g\left|u_{n}\right|^{r+1} d x+o(1)
$$

and

$$
\mathcal{J}_{\lambda, M}\left(u_{n}\right)=a\left(\frac{1}{p}-\frac{1}{r+1}\right)\left\|u_{n}\right\|_{X_{0}}^{p}+b\left(\frac{1}{p^{2}}-\frac{1}{r+1}\right)\left\|u_{n}\right\|_{X_{0}}^{p^{2}}+o(1),
$$

which contradicts $\mathcal{J}_{\lambda, M}\left(u_{n}\right) \rightarrow c_{\lambda}<0$ as $n \rightarrow \infty$. Furthermore,

$$
o(1)=\left\langle\mathcal{J}_{\lambda, M}^{\prime}\left(u_{n}\right), \phi\right\rangle=\left\langle\mathcal{J}_{\lambda, M}^{\prime}\left(u_{\lambda}^{+}\right), \phi\right\rangle+o(1) \quad \text { for all } \phi \in X_{0}
$$

Thus, $u_{\lambda}^{+} \in \mathcal{N}_{\lambda, M}(\Omega)$ is a nonzero solution of (1.1) and $\mathcal{J}_{\lambda, M}\left(u_{\lambda}^{+}\right) \geq c_{\lambda}$. Next, we will prove that $\mathcal{J}_{\lambda, M}\left(u_{\lambda}^{+}\right)=c_{\lambda}$. Since

$$
\begin{aligned}
\mathcal{J}_{\lambda, M}\left(u_{\lambda}^{+}\right)= & \frac{a}{p}\left\|u_{\lambda}^{+}\right\|_{X_{0}}^{p}+\frac{b}{p^{2}}\left\|u_{\lambda}^{+}\right\|_{X_{0}}^{p^{2}}-\frac{\lambda}{q+1} \int_{\Omega} f\left|u_{\lambda}^{+}\right|^{q+1} d x-\frac{1}{r+1} \int_{\Omega} g\left|u_{\lambda}^{+}\right|^{r+1} d x \\
= & \left(\frac{a}{p}-\frac{a}{r+1}\right)\left\|u_{\lambda}^{+}\right\|_{X_{0}}^{p}+\left(\frac{b}{p^{2}}-\frac{b}{r+1}\right)\left\|u_{\lambda}^{+}\right\|_{X_{0}}^{p^{2}} \\
& +\left(\frac{\lambda}{r+1}-\frac{\lambda}{q+1}\right) \int_{\Omega} f\left|u_{\lambda}^{+}\right|^{q+1} d x \\
\leq & \lim _{n \rightarrow \infty}\left[\left(\frac{a}{p}-\frac{a}{r+1}\right)\left\|u_{n}\right\|_{X_{0}}^{p}+\left(\frac{b}{p^{2}}-\frac{b}{r+1}\right)\left\|u_{n}\right\|_{X_{0}}^{p^{2}}\right. \\
& \left.+\left(\frac{\lambda}{r+1}-\frac{\lambda}{q+1}\right) \int_{\Omega} f\left|u_{n}\right|^{q+1} d x\right] \\
= & \lim _{n \rightarrow \infty} \inf _{\mathcal{J}_{\lambda, M}\left(u_{n}\right)=c_{\lambda} .}
\end{aligned}
$$

Hence, $\mathcal{J}_{\lambda, M}\left(u_{\lambda}^{+}\right)=c_{\lambda}$. Moreover, we have $u_{\lambda}^{+} \in \mathcal{N}_{\lambda, M}^{+}(\Omega)$. In fact, if $u_{\lambda}^{+} \in \mathcal{N}_{\lambda, M}^{-}(\Omega)$, by Lemma 2.4, there are unique $t^{+}$and $t^{-}$such that $t^{+} u_{\lambda}^{+} \in \mathcal{N}_{\lambda, M}^{+}(\Omega)$ and $t^{-} u_{\lambda}^{+} \in \mathcal{N}_{\lambda, M}^{-}(\Omega)$, we have $t_{\lambda}^{+}<t_{\lambda}^{-}=1$. Since

$$
\frac{d}{d t} \mathcal{J}_{\lambda, M}\left(t_{\lambda}^{+} u_{\lambda}^{+}\right)=0 \quad \text { and } \quad \frac{d^{2}}{d t^{2}} \mathcal{J}_{\lambda, M}\left(t_{\lambda}^{+} u_{\lambda}^{+}\right)>0
$$

there exists $t_{\lambda}^{+}<t^{*} \leq t_{\lambda}^{-}$such that $\mathcal{J}_{\lambda, M}\left(t_{\lambda}^{+} u_{\lambda}^{+}\right)<\mathcal{J}_{\lambda, M}\left(t^{*} u_{\lambda}^{+}\right)$. By Lemma 2.4, we get

$$
\mathcal{J}_{\lambda, M}\left(t_{\lambda}^{+} u_{\lambda}^{+}\right)<\mathcal{J}_{\lambda, M}\left(t^{*} u_{\lambda}^{+}\right) \leq \mathcal{J}_{\lambda, M}\left(t_{\lambda}^{-} u_{\lambda}^{+}\right)=\mathcal{J}_{\lambda, M}\left(u_{\lambda}^{+}\right)
$$

which is a contradiction. Since $\mathcal{J}_{\lambda, M}\left(u_{\lambda}^{+}\right)=\mathcal{J}_{\lambda, M}\left(\left|u_{\lambda}^{+}\right|\right)$and $\left|u_{\lambda}^{+}\right| \in \mathcal{N}_{\lambda, M}^{+}(\Omega)$, we see that $u_{\lambda}^{+}$ is a solution of (1.1) by Lemma 2.3. 
Similarly, we can obtain the theorem of existence of a local minimum for $\mathcal{J}_{\lambda, M}$ on $\mathcal{N}_{\lambda, M}^{-}(\Omega)$ as follows.

Theorem 3.5 Assume that (H1)-(H4) hold. For each $0<\lambda<\Gamma_{0}\left(\Gamma_{0}\right.$ is as in Lemma 3.3), the functional $\mathcal{J}_{\lambda, M}$ has a minimizer $u_{\lambda}^{-}$in $\mathcal{N}_{\lambda, M}^{-}(\Omega)$ satisfying:

(1) $\mathcal{J}_{\lambda, M}\left(u_{\lambda}^{-}\right)=c_{\lambda}^{-}$;

(2) $u_{\lambda}^{-}$is a solution of (1.1).

Finally, we give the main result of this paper as follows.

Theorem 3.6 Suppose that the conditions (H1)-(H4) hold. Then there exists $\Gamma_{0}>0$ such that for $\lambda \in\left(0, \Gamma_{0}\right),(1.1)$ has at least two solutions.

Proof From Theorems 3.4, 3.5, we see that (1.1) has two solutions $u_{\lambda}^{+}$and $u_{\lambda}^{-}$such that $u_{\lambda}^{+} \in \mathcal{N}_{\lambda, M}^{+}(\Omega), u_{\lambda}^{-} \in \mathcal{N}_{\lambda, M}^{-}(\Omega)$. Since $\mathcal{N}_{\lambda, M}^{+}(\Omega) \cap \mathcal{N}_{\lambda, M}^{-}(\Omega)=\emptyset$, we see that $u_{\lambda}^{+}$and $u_{\lambda}^{-}$are different.

Remark 3.2 Obviously, if $p=2$, then (H3) and (H4) hold. Moreover, if $p=2, s=1, a=1$, and $b=0$, then Theorem 3.6 is in agreement with Theorem 1.2 in [1].

\section{Competing interests}

The author declares that he has no competing interests.

Author's contributions

All results belong to $C B$.

\section{Acknowledgements}

This work is supported by Natural Science Foundation of China (11571136 and 11271364).

Received: 5 August 2016 Accepted: 11 November 2016 Published online: 25 November 2016

\section{References}

1. Wu, TF: Multiplicity results for a semilinear elliptic equation involving sign-changing weight function. Rocky Mt. J. Math. 39, 995-1011 (2009)

2. Autuori, G, Fiscella, A, Pucci, P: Stationary Kirchhoff problems involving a fractional elliptic operator and a critical nonlinearity. Nonlinear Anal. 125, 699-714 (2015)

3. Chen, $\mathrm{CY}, \mathrm{Kuo}, \mathrm{YC}, \mathrm{Wu}$, TF: The Nehari manifold for a Kirchhoff type problem involving sign-changing weight functions. J. Differ. Equ. 250, 1876-1908 (2011)

4. Fiscella, A, Valdinoci, E: A critical Kirchhoff type problem involving a nonlocal operator. Nonlinear Anal. 94, 156-170 (2014)

5. Pucci, P, Saldi, S: Critical stationary Kirchhoff equations in $\mathbb{R}^{N}$ involving nonlocal operators. Rev. Mat. Iberoam. 32, 1-22 (2016)

6. Pucci, P, Xiang, M, Zhang, B: Multiple solutions for nonhomogeneous Schrödinger-Kirchhoff type equations involving the fractional $p$-Laplacian in $\mathbb{R}^{N}$. Calc. Var. Partial Differ. Equ. 54(3), 2785-2806 (2015)

7. Mishra, PK, Sreenadh, K: Existence and multiplicity results for fractional $p$-Kirchhoff equation with sign changing nonlinearities. Adv. Pure Appl. Math. (2015). doi:10.1515/apam-2015-0018

8. Di Nezza, E, Palatucci, G, Valdinoci, E: Hitchhiker's guide to the fractional Sobolev spaces. Bull. Sci. Math. 136, 521-573 (2012)

9. Drabek, P, Pohozaev, SI: Positive solutions for the $p$-Laplacian: application of the fibering method. Proc. R. Soc. Edinb. A 127, 703-726 (1997)

10. Ni, WM, Takagi, I: On the shape of least energy solution to a Neumann problem. Commun. Pure Appl. Math. 44, 819-851 (1991)

11. Ekeland, I: On the variational principle. J. Math. Anal. Appl. 17, 324-353 (1974) 